\title{
Demographic, medical and visual aspects of Dia- betic Retinopathy (DR) and Diabetic Macular Ede- ma (DME) in South African diabetic patients*
}

\author{
Anusha Y Sukha ${ }^{\mathrm{a}}$ and Alan Rubin ${ }^{\mathrm{b}}$ \\ a,b Department of Optometry, University of Johannesburg, PO Box 524, Auckland Park, 2006 South \\ Africa
}

Received 13 January 2009; revised version accepted 12 March 2009

\begin{abstract}
Objective: To investigate some of the demographic, medical, and visual aspects of diabetic retinopathy (DR) and diabetic macula edema (DME) in diabetics attending an urban clinic in Johannesburg, Gauteng.

Design: In this cross-sectional study, 202 diabetic patients were recruited. Demographic variables included age, gender, race, age of diagnosis, duration of diabetes mellitus (DM), and social habits. Medical variables included systemic conditions present, blood pressures, body mass indices (BMI), lipid profiles, glycerated haemoglobin (HbAlc), and other biochemical data. Visual variables included distance, pinhole and near visual acuities, contrast visual acuities (CVA), refractive status, colour vision, central visual field evaluation with the Amsler grid, intraocular pressures (IOP), fundus photography and administration of the Impact of Visual Impairment (IVI) questionnaire. All variables were compared between diabetic subjects with and without DR and DME in both right and left eyes.

Results: Overall prevalence of DR was $22.8 \%$ and DME $12.5 \%$. In DR subjects, significant results indicated that Whites were more likely to present with DR $(p=0.002)$. Subjects with DR had a high-
\end{abstract}

er mean duration of DM ( $p=0.002)$ and a higher mean diastolic blood pressure $(p=0.035)$. Autorefraction suggested that more myopia and less astigmatism might be associated with DR. A higher mean CVA at the $2.5 \%$ level in DR was significant in both the right eyes $(p=0.042)$ and left eyes $(p=$ 0.035 ). These subjects also reported a higher mean IVI score in the consumer and social interaction domain $(p=0.032)$. Similarly, DME subjects displayed a higher mean duration of DM $(p=0.042)$ and a higher mean diastolic blood pressure $(p=$ 0.048). A higher mean CVA was associated at both the $10 \%$ level: right eyes ( $p=0.021)$; and left eyes $(p=0.046)$, and at the $2.5 \%$ level: right eyes ( $p=$ $0.033)$ and left eyes $(p=0.045)$. A higher mean IVI score in leisure and work $(p=0.026)$, consumer and social interactions ( $p=0.01)$ and emotional reaction to vision loss $(p=0.018)$ was reported in subjects with DME.

Conclusion: This study has identified possible demographic, medical and visual risk factors of DR and DME in South African diabetic patients.

Key words: diabetes, diabetic retinopathy, diabetic macular edema, contrast visual acuity, diabetic demographics

\footnotetext{
*This paper is based on research by AY Sukha towards a Masters degree in the Department of Optometry of the University of Johannesburg with the supervision of Professor A Rubin
}

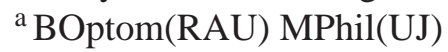

${ }^{\mathrm{b}}$ DipOptom CAS(NewEnCO) MPhil(RAU) DPhil(RAU) 


\section{Introduction}

Diabetic retinopathy (DR) has become the leading cause of visual impairment and blindness among the working age group in both developed and developing countries $^{1}$, and it is now probably the most frequent manifestation of a systemic disease encountered in primary-care institutions. Optometrists and ophthalmologists form part of the multi-disciplinary team aiding diabetic care. They are also often the first to examine patients with undiagnosed diabetes, or early and possibly asymptomatic DR or even $\mathrm{DME}^{2,3}$. This possibly is even more so in South Africa, where optometrists are probably the most accessible eye care professionals to the public. General hospitals, clinics, and non-government organizations providing eye care are limited (approximately 25 government clinics to cater for 32 million people) and have very few optometrists and ophthalmologists available ${ }^{4}$.

The need for early detection of DR and DME is further highlighted by the development of new therapies. Currently medical interventions include laser photocoagulation and vitrectomy that only target advanced DR and DME with variable success. Potential pharmacological therapies are now being developed to target the underlying biochemical mechanisms that cause DR and DME. The aim of these therapies is to find pharmacological agents that slow down the progression of DR and DME, in their early stages ${ }^{5,6}$. With the introduction of these therapies in the future, reliable procedures will be needed to detect early DR and DME. However, due to the insidious nature of $\mathrm{DM}$, the asymptomatic stages of DR and DME are not easily detectable by fundus photography, ophthalmoscopy, fluorescein angiography, or even optical coherence tomography ${ }^{5,7}$. Nevertheless, there is evidence that during these early stages alterations occur in the retinal ganglion cells and inner retinal neurons which in turn causes changes, for example, in colour vision and contrast sensitivity ${ }^{7}$. Therefore, this study was principally aimed at identifying and understanding some visual aspects of DR and DME such as contrast visual acuities, refractive behaviour, and colour vision. But demographic and medical profiles were also investigated to provide a more holistic understanding of this complicated disease.

\section{Methods}

Two hundred and two subjects $(N=202)$ attending the diabetic clinic at the Helen Joseph hospital in
Johannesburg, Gauteng, South Africa were randomly recruited into the study. Participation was voluntary, and all the necessary ethical clearances for the study were obtained from The University of Witwatersrand (who administer the clinic) and the University of Johannesburg. Demographic and medical data were recorded on the day of the examinations. Age was defined as the age of the patient at the time of examination, and age of diagnosis of DM as the time the diagnosis was first recorded by a physician on the patient's hospital record card. The duration of DM was calculated as the period between the age of diagnosis and the age at the time of the examination. For the purposes of data analysis, patients were classified by sample size, from greatest to least, into their respective ethnic groups, namely Coloured, Black, Indian or White. Biochemical results within three months of the visual examination were used for analyses. Optometrists at different stations conducted the visual assessments. Three refractive state measurements per eye were measured using the Huvitz MRK 3100P autorefractor. The instrument rounded measurements to $0.25 \mathrm{D}$, and the matrix averages of the measurements per eye were used for further data analysis. Monocular habitual distance acuity and pinhole visual acuity was measured using the Lighthouse ETDRS charts. Contrast visual acuity (CVA) results were obtained using the logarithmic new ETDRS chart from Precision Vision, at contrast levels $100 \%, 10 \%$ and $2.5 \%$. Testing distances included 4 metres, or at 2 and 1 metre where required. The letter scoring method and the manipulation of the logarithmic scale into smaller denominations were used to record all acuities as it provided greater accuracy. (For example, acuity of $4 / 20-2$ equals letter score of $65-2$, which is 63 , and 63 converted to LogMAR notation equates to 0.74.) Colour vision assessments in the subjects were performed to determine whether the Farnsworth D15 colour vision tests would be feasible as a screening test to differentiate between subjects with and without DR and DME. An average of three intra-ocular pressure measurements per eye was obtained using a Reichert NCT II non-contact tonometer. Central visual field abnormalities were recorded via an Amsler Grid consisting of white lines making up $5 \mathrm{~mm}$ squares on a black background. A non-mydriatic $45^{\circ}$ retinal camera, the Topcon TRC-NW200 was used to obtain fundus images, centering on the macula. The design of this camera allowed for images to be ob- 
tained through pupils as small as $3.7 \mathrm{~mm}$ and pupil dilation was encouraged by allowing each subject to spend five minutes in a dark room before photography. Image quality was monitored with periodic feedback to the photographers, and if necessary more than one photograph was taken. The digital images were then evaluated independently by two experienced ophthalmologists, and diagnosed in accordance with the International Clinical Disease Severity Scale ${ }^{8}$. Lastly, the IVI 32-item questionnaire was administered to measure the impact of vision impairment in the following five domains of functioning: leisure and work, consumer and social interactions, household and personal care, mobility, and emotional reaction to vision loss. Responses to items were rated from not at all (0), rarely (1), a little (2), a fair amount (3), and a lot (4), all the time or cannot do because of eyesight (5), and do not do because of other reasons (8). Total and domain scores are an arithmetic average of the rating where an item rate 8 is not included in the final score ${ }^{9}$. The reason for this choice of questionnaire was that it had been reported to have sufficient internal and construct validity and because the questionnaire yielded consistent results between interviewers over time, it has the potential to evaluate outcomes of interventions $^{9,10}$. Subject identity, diabetes status, and all other clinical and medical factors were masked and unknown to the ophthalmologists, photographers and optometrists.
Data analysis was done using the Statistical Package for Social Sciences (SPSS) software provided by the Statistical Consulting Department of the University of Johannesburg, and multivariate analysis was performed on autorefraction measurements, using software developed by Harris, Rubin and Malan from the Department of Optometry of the University of Johannesburg. Parametric two tailed $t$-tests and chi-squared tests were used to obtain significant differences when all variables were compared between subjects with and without DR and between subjects with and without DME in right and left eyes.

\section{Results}

\section{Demographic and Medical Results}

Table 1 represents a comparison of the descriptive variables between diabetic subjects with and without DR and DME.

The prevalence of DR and DME are presented in Figure 1. The overall prevalence of DR was calculated at 22.8\% $(n=46)$ and DME at $12.4 \%(n=$ 25 ). Both DR and DME were equally prevalent in both Type 1 and Type $2 \mathrm{DM}$. These results were also comparable to that for the whole sample. A possible explanation for this finding may be attributed to the standard of care in these subjects. A majority subjects at this clinic are diagnosed with DM in an early stage and thereafter assessed regularly (every 3 months).

Table 1. A comparison of descriptive statistics between subjects without DR to those with DR and in subjects without DME to those with DME. Categorical variables are represented with frequencies (f) and percentages (\%). Continuous variables are represented with means (M), and standard deviations (SD). The sub-groups of sample sizes are represented by $n$. Systemic conditions and social habits represent subjects who responded “yes”. Recommended control values are indicated in brackets next to the appropriate biochemical test. Significant results are indicated in bold type.

\begin{tabular}{|c|c|c|c|c|c|c|}
\hline & & & No DR $(n=156)$ & DR $(n=46)$ & $\begin{array}{l}\text { No DME } \\
(n=177)\end{array}$ & DME $(n=25)$ \\
\hline & & $n$ & $f(\%)$ & $f(\%)$ & f $(\%)$ & f $(\%)$ \\
\hline \multicolumn{7}{|l|}{ Gender } \\
\hline & Male & 75 & $59(37.8)$ & $16(34.8)$ & $64(36.2)$ & $11(44)$ \\
\hline & Female & 127 & $97(62.2)$ & $30(65.2)$ & $11(44)$ & $14(56)$ \\
\hline \multicolumn{7}{|l|}{ Race } \\
\hline & Black & 74 & $66(42.3)$ & $8(17.4)$ & $68(38.4)$ & $6(24)$ \\
\hline & Coloured & 88 & $65(41.7)$ & $23(50)$ & $78(44.1)$ & $10(40)$ \\
\hline & Indian & 20 & $15(9.6)$ & $5(10.9)$ & $17(9.6)$ & $3(12)$ \\
\hline & White & 20 & $10(6.4)$ & $10(21.7)$ & $14(7.9)$ & $6(24)$ \\
\hline \multirow{2}{*}{\multicolumn{7}{|c|}{ Type of DM }} \\
\hline & & & & & & \\
\hline & Type 1 DM & 123 & $95(60.9)$ & $28(60.9)$ & $108(61)$ & $15(60)$ \\
\hline & Type 2 DN & 179 & $61(39.1)$ & $18(39.1)$ & $69(39)$ & $10(40)$ \\
\hline
\end{tabular}




\begin{tabular}{|c|c|c|c|c|c|}
\hline & & $M / S D$ & $M / S D$ & $M / S D$ & $M / S D$ \\
\hline Age (years) & & $51.2 \pm 14.5$ & $55 \pm 12.3$ & $51.6 \pm 14$ & $55 \pm 13$ \\
\hline Age Of Diagnosis (years) & & $41 \pm 13$ & $41 \pm 13$ & $41 \pm 13$ & $40.1 \pm 14$ \\
\hline Duration (years) & & $10 \pm 9.6$ & $13.7 \pm 9$ & $10.3 \pm 9.8$ & $14.5 \pm 7.8$ \\
\hline Body Mass Index $\left(\mathrm{kg} / \mathrm{m}^{2}\right)$ & & $30.4 \pm 7$ & $31.7 \pm 7$ & $30.8 \pm 7$ & $29.9 \pm 6.7$ \\
\hline Systolic BP ( $140 \mathrm{mmHg}$ ) & & $134.8 \pm 21$ & $138 \pm 18$ & $135.4 \pm 20,7$ & $136.7 \pm 19$ \\
\hline Diastolic BP ( $84 \mathrm{mmHg})$ & & $80 \pm 11$ & $84.3 \pm 12.5$ & $80.5 \pm 11$ & $85.4 \pm 13$ \\
\hline & & & & & \\
\hline Systemic Conditions & $n$ & $f(\%)$ & f $(\%)$ & $f(\%)$ & $f(\%)$ \\
\hline Hypertension & 134 & $98(62.8)$ & $36(78.3)$ & $115(65)$ & $19(76)$ \\
\hline Renal & 7 & $6(3.8)$ & $1(2.2)$ & $5(2.8)$ & $2(8)$ \\
\hline Cardiovascular Disease & 36 & $26(16.7)$ & $10(21.7)$ & $29(16.4)$ & $7(28)$ \\
\hline Respiratory & 8 & $8(5.1)$ & 0 & $8(4.5)$ & 0 \\
\hline Central Nervous System Disorders & 9 & $6(3.8)$ & $3(6.5)$ & $8(4.5)$ & $1(4)$ \\
\hline & & & & & \\
\hline \multicolumn{6}{|l|}{ Social Habits } \\
\hline Alcohol & 24 & $18(11.5)$ & $6(13)$ & $22(12.4)$ & $2(8)$ \\
\hline Drugs & 1 & $1(0.6)$ & 0 & $1(0.6)$ & 0 \\
\hline Smoking & 40 & $35(22.4)$ & $5(10.9)$ & $39(22)$ & $1(4)$ \\
\hline Diet & 145 & $110(70.5)$ & $35(76.1)$ & $125(70.6)$ & $20(80)$ \\
\hline Diaghomingl Poculto & & & Gen & & $M / C D$ \\
\hline $\begin{array}{l}\text { Biochemical Results } \\
\text { HBA1C }(<6-<7 \%)\end{array}$ & & $\frac{M / S D}{10 \pm 3.5}$ & $\frac{M / S D}{10 \pm 2.8}$ & $\frac{M / S D}{9.9 \pm 3.4}$ & $\begin{array}{l}M / S D \\
10.4 \pm 2.95\end{array}$ \\
\hline UeSodium (135-147 mmol/l) & & $137 \pm 11.5$ & $138 \pm 3$ & $137 \pm 10.7$ & $137.7 \pm 3$ \\
\hline Potassium (3.3-5.3 mmol/l) & & $4 \pm 0.5$ & $4 \pm 0.5$ & $4 \pm 0.5$ & $4.2 \pm 0.5$ \\
\hline Chloride $(99-113 \mathrm{mmol} / \mathrm{l})$ & & $108 \pm 10,4$ & $108 \pm 10$ & $107.8 \pm 9.8$ & $108.6 \pm 13.7$ \\
\hline $\mathrm{CO} 2(18-29 \mathrm{mmol} / \mathrm{I})$ & & $25.6 \pm 3.6$ & $25 \pm 3.2$ & $25.6 \pm 3.4$ & $24.4 \pm 4$ \\
\hline Urea $(2.8-7.0 \mathrm{mmol} / \mathrm{l})$ & & $6.5 \pm 7.9$ & $5.5 \pm 2.6$ & $6.1 \pm 7$ & $7.8 \pm 5.9$ \\
\hline Creatinine $(60-100 \mathrm{mmol} / \mathrm{l})$ & & $86.7 \pm 44$ & $78.7 \pm 23.9$ & $82.8 \pm 35$ & $101.8 \pm 68$ \\
\hline Total Cholestrol $(<5.0 \mathrm{mmol} / \mathrm{l})$ & & $4.75 \pm 1.2$ & $4.9 \pm 1.4$ & $4.8 \pm 1.1$ & $4.9 \pm 1.6$ \\
\hline $\mathrm{HDL}(>1.2 \mathrm{mmol} / \mathrm{l})$ & & $1 \pm 0.7$ & $1 \pm 0.8$ & $1 \pm 0.8$ & $0.9 \pm 0.3$ \\
\hline $\mathrm{LDL}(<3.0 \mathrm{mmol} / \mathrm{I})$ & & $2.9 \pm 1$ & $2.8 \pm 1.2$ & $2.9 \pm 1$ & $3 \pm 1.3$ \\
\hline Triglycerides (<1.5 mmol/l) & & $1.8 \pm 1.3$ & $2 \pm 1.4$ & $1.9 \pm 1.3$ & $2 \pm 1.6$ \\
\hline \multirow{2}{*}{\multicolumn{6}{|c|}{ Visual Assesment }} \\
\hline & & & & & \\
\hline \multicolumn{6}{|l|}{ Autorefraction } \\
\hline Right Eye & & $-0.06 /-0.12 \times 105$ & $-0.52 /-0.10 \times 89$ & $-0.16 /-0.14 \times 99$ & $-0.23 /-0.26 \times 123$ \\
\hline Left Eye & & $-0.14 /-0.24 \times 117$ & $-0.50 /-0.40 \times 6$ & $-0.24 /-0.22 \times 119$ & $-0.32 /-0.89 \times 19$ \\
\hline Distance VA (LogMar) & & $M / S D$ & $M / S D$ & $M / S D$ & $M / S D$ \\
\hline Right Eye & & $0.21 \pm 0.32$ & $0.31 \pm 0.31$ & $0.22 \pm 0.3$ & $0.35 \pm 0.37$ \\
\hline Left Eye & & $0.24 \pm 0.36$ & $0.36 \pm 0.45$ & $0.25 \pm 0.38$ & $0.39 \pm 0.42$ \\
\hline \multicolumn{6}{|l|}{ Pinhole VA } \\
\hline Right Eye & & $0.16 \pm 0.3$ & $0.26 \pm 0.34$ & $0.17 \pm 0.3$ & $0.29 \pm 0.4$ \\
\hline \begin{tabular}{|l} 
Left Eye \\
\end{tabular} & & $0.18 \pm 0.37$ & $0.33 \pm 0.52$ & $0.19 \pm 0.38$ & $0.4 \pm 0.6$ \\
\hline \multicolumn{6}{|l|}{ Contrast Sensitivity - Right Eye } \\
\hline $100 \%$ & & $0.34 \pm 0.33$ & $0.36 \pm 0.31$ & $0.3 \pm 0.32$ & $0.42 \pm 0.36$ \\
\hline
\end{tabular}




\begin{tabular}{|c|c|c|c|c|c|}
\hline $10 \%$ & & $0.55 \pm 0.41$ & $0.63 \pm 0.42$ & $0.54 \pm 0.4$ & $0.75 \pm 0.46$ \\
\hline $2.50 \%$ & & $0.92 \pm 0.55$ & $1.1 \pm 0.6$ & $0.93 \pm 0.56$ & $1.2 \pm 0.6$ \\
\hline \multicolumn{6}{|l|}{ Contrast Sensitivity - Left Eye } \\
\hline $100 \%$ & & $0.35 \pm 0.37$ & $0.44 \pm 0.46$ & $0.36 \pm 0.4$ & $0.46 \pm 0.42$ \\
\hline $10 \%$ & & $0.57 \pm 0.42$ & $0.71 \pm 0.48$ & $0.58 \pm 0.42$ & $0.76 \pm 0.5$ \\
\hline \multirow[t]{2}{*}{$2.50 \%$} & & $0.94 \pm 0.56$ & $1.16 \pm 0.64$ & $0.96 \pm 0.58$ & $1.2 \pm 0.6$ \\
\hline & $n$ & $f(\%)$ & f $(\%)$ & $f(\%)$ & $f(\%)$ \\
\hline Colour Vision & & & & $1+2$ & (n) \\
\hline \multirow[t]{4}{*}{ Right Eye } & 33 & $20(12.8)$ & $13(28.3)$ & $26(14.7)$ & $7(28)$ \\
\hline & 152 & $123(78.8)$ & $29(63)$ & $137(77.4)$ & $15(60)$ \\
\hline & 12 & $10(6.4)$ & $2(4.3)$ & $11(6.2)$ & $1(4)$ \\
\hline & 5 & $3(1.9)$ & $2(4.3)$ & $3(1.7)$ & $5(2.5)$ \\
\hline \multicolumn{6}{|l|}{ Left Eye } \\
\hline No Errors & 32 & $22(14.1)$ & $10(21.7)$ & $26(14.7)$ & $6(24)$ \\
\hline Tritan & 153 & $120(76.9)$ & $33(71.1)$ & $137(77.4)$ & $16(64)$ \\
\hline Deutan & 6 & $5(3.2)$ & $1(2.2)$ & $5(2.8)$ & $1(4)$ \\
\hline Protan & 9 & $7(4.5)$ & $2(4.3)$ & $7(4)$ & $2(8)$ \\
\hline \multirow{2}{*}{\multicolumn{6}{|c|}{ Amsler Grid }} \\
\hline & & & & & \\
\hline \multirow[t]{2}{*}{ Right Eye } & 159 & $123(78.8)$ & $36(78.3)$ & $140(79.1)$ & $19(76)$ \\
\hline & 42 & $32(20.5)$ & $10(21.7)$ & $36(20.3)$ & $6(24)$ \\
\hline \multirow{4}{*}{$\begin{array}{l}\text { No Defects } \\
\text { Defects }\end{array}$} & 155 & $119(76.3)$ & $33(21.2)$ & $135(76.3)$ & $20(80)$ \\
\hline & 43 & $33(21.2)$ & $10(21.7)$ & $38(21.5)$ & $5(20)$ \\
\hline & & & & & \\
\hline & $n$ & $M / S D$ & $M / S D$ & $M / S D$ & $M / S D$ \\
\hline \multicolumn{6}{|l|}{ Intra Ocular Pressure $(\mathrm{mmHg})$} \\
\hline Right Eye & & $17.54 \pm 4.9$ & $16.8 \pm 4.4$ & $17.62 \pm 4.9$ & $15.62 \pm 4.1$ \\
\hline \multirow[t]{2}{*}{ Left Eye } & & $17.88 \pm 5$ & $17,41 \pm 4,12$ & $17.92 \pm 4.9$ & $16.7 \pm 3.9$ \\
\hline & $n$ & f $(\%)$ & $f(\%)$ & $f(\%)$ & $f(\%)$ \\
\hline & & & & & \\
\hline \multicolumn{6}{|l|}{ DR in either right or left eyes } \\
\hline \multicolumn{2}{|l|}{ 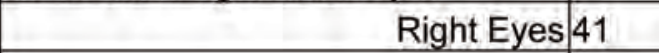 } & & & $22(12.4)$ & $19(76)$ \\
\hline \multirow[t]{2}{*}{ Left Eyes } & 41 & & & $20(11.3)$ & $21(84)$ \\
\hline & & & & & \\
\hline \multicolumn{6}{|l|}{ DME in either right or left eyes } \\
\hline \multicolumn{2}{|l|}{$\begin{array}{r}\text { Right Eyes } 21 \\
\end{array}$} & $2(1.3)$ & $19(41.3)$ & & \\
\hline \multirow[t]{3}{*}{ Left Eyes } & 20 & $1(0.6)$ & $19(41.3)$ & & \\
\hline & & & & & \\
\hline & $n$ & $M / S D$ & $M / S D$ & $M / S D$ & $M / S D$ \\
\hline \multicolumn{6}{|l|}{ Activities Of Daily Living } \\
\hline Leisure and work & & $3.5 \pm 4.6$ & $4.5 \pm 5$ & $3.5 \pm 4.4$ & $6.04 \pm 5.8$ \\
\hline Consumer and social interactions & & $3.9 \pm 4.8$ & $5.6 \pm 4.9$ & $4 \pm 4.8$ & $6.3 \pm 4.7$ \\
\hline Household and personal care & & $2.7 \pm 3.9$ & $3.8 \pm 3.9$ & $2.8 \pm 4$ & $4.2 \pm 3.7$ \\
\hline Mobility & & $6,7 \pm 7,8$ & $8.7 \pm 9.9$ & $6.7 \pm 8$ & $9.8 \pm 9$ \\
\hline Emotional reaction to vision loss & & $7.7 \pm 7$ & $10.2 \pm 8.6$ & $7.6 \pm 7$ & $12.6 \pm 9.5$ \\
\hline Last Eye Exam (months) & & $21 \pm 26$ & $24 \pm 31$ & $20.7 \pm 25.5$ & $29.4 \pm 37.8$ \\
\hline
\end{tabular}


Blood sugar levels are also monitored at each visit, and where necessary medications are altered to maintain stricter control of their HBA1c. A further explanation that might contribute to these findings are, that the average blood pressure and cholesterol levels in this group of subjects were within recommended control ranges, and these parameters if uncontrolled are found to increase the prevalence and progression of DR and DME. Hence, the current results may suggest that in a group of early diagnosed, and regularly monitored and controlled diabetics, the prevalence of
DR and DME may be similar.

Gender comparisons showed no statistically significant differences between the absence or presence of DR and DME, even though female subjects had a greater occurrence of DR and DME. Ethnic comparison showed higher prevalence of DR in White subjects, significant at $5 \%$ level $(p=0.002, \varnothing=0.273)$, and DME at $10 \%(p=0.66, \varnothing=0.189)$. Hence, there are indications that the White diabetic person is more likely to present with DR and DME, and the Black diabetic person least likely.

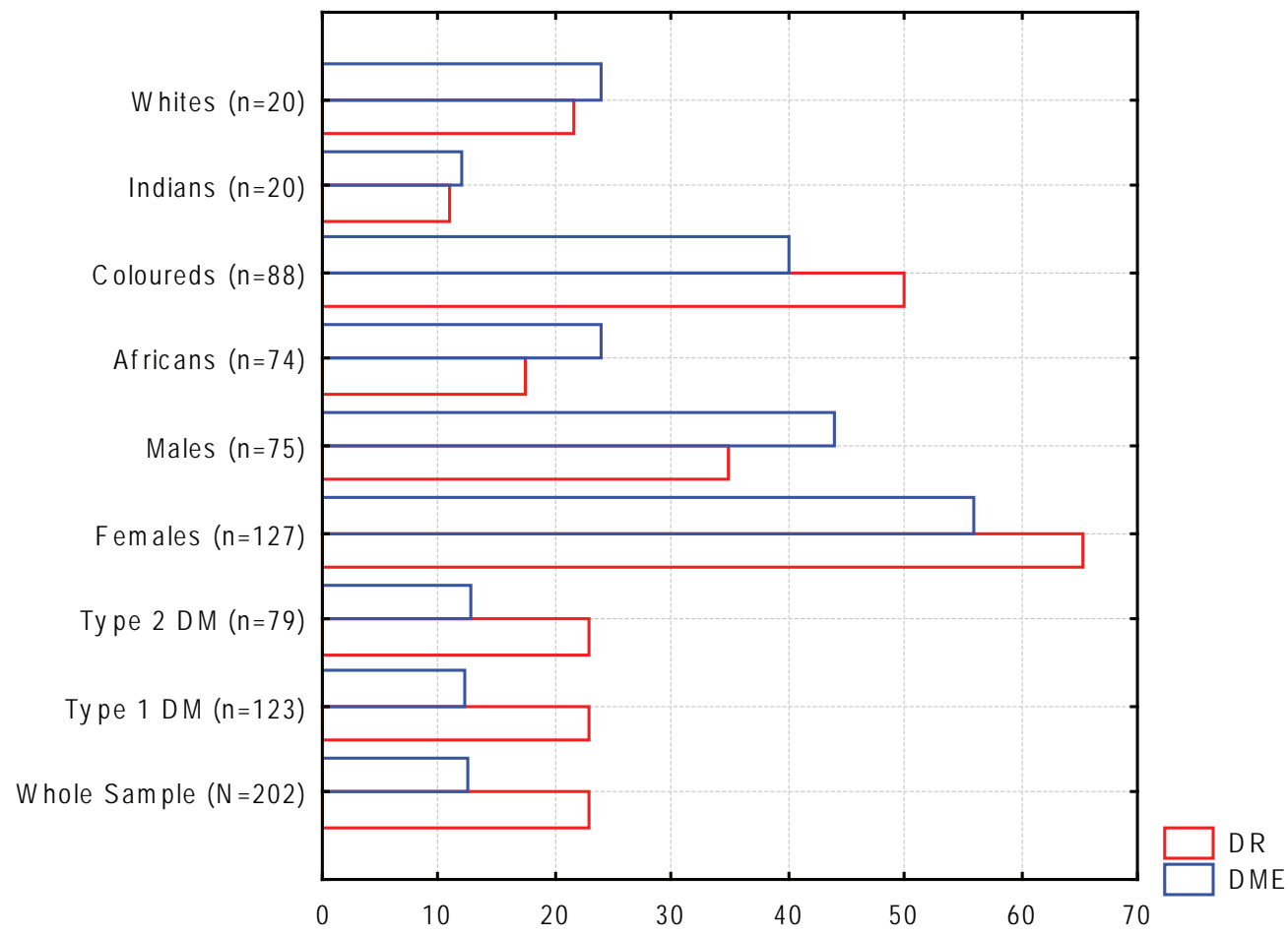

Figure 1. Categorized bar chart representing percentage prevalence of DR and DME in either right, left or both eyes for all diabetic subjects $(N=202)$.

The mean age and age of diagnosis between these subjects (DR and DME) showed no significant differences. As expected, the duration of DM revealed significant differences in both DR $(t(197)=-2.302$, $p=0.022)$ and in DME $(t(197)=-2.046, p=0.042)$ between subjects. This indicates that on average subjects who have a longer duration of DM, are more likely to present with DR and DME. Results also indicate that BMI and blood pressures are comparable between the DR and DME groups. Of these only mean diastolic blood pressure was significantly higher in subjects with DR $(t(188)=-2.120, p=0.035)$ and DME $(t(188)=-1.989, \mathrm{p}=0.048)$.

Of the systemic conditions both hypertension and cardiovascular diseases were more frequent in sub- jects with DR and DME. However, the results only indicated hypertension in the DR group significant at a $10 \%$ level ( $p=0.051, \varnothing=0.137)$. All social habits and available biochemical results of subjects showed no statistically significant differences in this sample.

\section{Visual Results}

Autorefraction results were analysed using multivariate methods and software developed by Harris, Rubin and Malan (of the Optometric Science Research Group). Satisfactory statistical analyses, for example, calculations of means, variances and testing of hypotheses, with this type of data have only recently become possible based on transformation of dioptric power from clinical notation to a matrix rep- 
resentation. With matrices, Harris ${ }^{11-12}$ demonstrates that dioptric power can be ordered or ranked, squared, and that this approach allows determination of sample means, variances and covariances, hypothesis testing and appropriate scientific graphical representation of dioptric power. The graphical representation of the dioptric power matrix $\mathbf{F}$ is in the vector space (see Figure 2) known as symmetric dioptric power space s1-12. $^{11}$. Each point in the space is represented by a matrix F. With these matrices, powers can be plotted on a set of three mutually orthogonal axes, $F_{\mathrm{I}} \mathbf{I}, F_{\mathrm{J}} \mathbf{J}$ and $F_{\mathrm{K}} \mathbf{K}$ in the Euclidean 3-space so that a binocularly perceivable three-dimensional scatter plot is produced ${ }^{11}$. To

\section{Right Eyes}

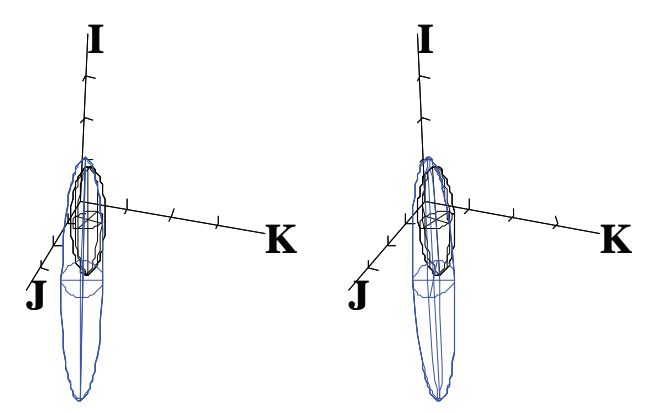

obtain the three-dimensional image, the two halves of the scatter plot must be fused by allowing the eyes to diverge slightly into an outward or exo-position relative to the plane of the $\operatorname{plot}^{11}$. The origin represents

the point of zero power, emmetropia or $0 \mathrm{D}$ in clinical notation. The axis $\left(F_{\mathrm{I}} \mathbf{I}\right)$ represents scalar powers, or spherical change; that is, whether eyes are myopic or hyperopic without astigmatism (having spherical or stigmatic ametropia), or being emmetropic. The ortho-antistigmatic power $\left(F_{\mathrm{J}} \mathbf{J}\right)$ represents all Jackson Crossed Cylinders or antistigmatic powers with axes in the vertical and horizontal meridians, $90^{\circ}$ and 180 ${ }^{\circ}$.The oblique antistigmatic $\left(F_{K} \mathbf{K}\right)$ axis represents all Left Eyes

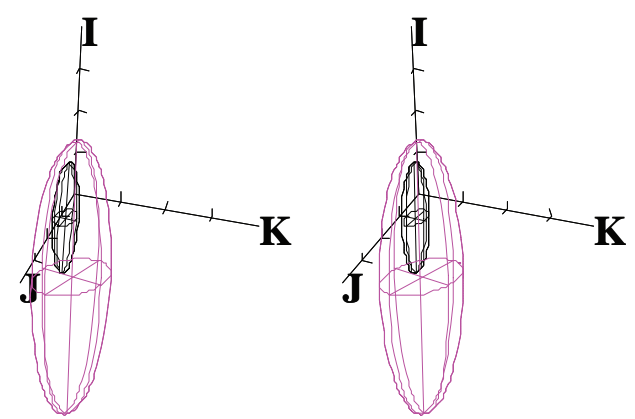

Figure 2. This stereo-pair scatter plot represents $95 \%$ confidence ellipsoids for autorefraction measurements. Blue (right eyes) and magenta (left eyes) ellipsoids represent diabetic subjects with DR and black ellipsoids those without DR. Thus, the presence of diabetic retinopathy (DR) was associated with more variation (larger ellipsoids) in refractive state and also a myopic shift (the centroids of the blue and magenta ellipsoids are shifted downwards relative to the black ellipsoids). In clinical terms, origins for both plots are emmetropia, that is, a refractive state of zero or $0 \mathrm{D}$. Tick intervals for the axes are $0.25 \mathrm{D}$. Each axis has four ticks (although only three appear on each axis with the fourth being at the end of the axis) and thus the axis lengths are each $1 \mathrm{D}$. Readers should allow their eyes to drift apart until a 3-dimensional percept is formed where the ortho-antistigmatic axis labeled $\mathbf{J}$, should extend downwards and towards the left. The spherical or stigmatic axis labeled I represents change from simple spherical hyperopia through emmetropia (the origin) to myopia (the negative half of axis I which is not shown in the stereo-pair). Each axis really extends from positive to negative infinity but only a small part of the positive half of each axis is shown above.

Jackson Crossed Cylinders with axes at $45^{\circ}$ and $135^{\circ}$. The estimated distribution of mean autorefraction measurements in subjects with and without DR was evaluated using 95\% confidence ellipsoids (ellipsoids centered on a sample mean ${ }^{12}$ ) in Figure 2. From the figure it can be seen that the centroids (or centres) of the blue and magenta ellipsoids are shifted downwards along the stigmatic axis (in comparison with the black ellipsoids) so for the samples with DR the means were slightly more myopic. Additionally, the blue and magenta ellipsoids are elongated along the stigmatic axis, so variations with (and, indeed without) DR are mainly stigmatic or spherical, that is, eyes vary by being more hyperopic or myopic mainly with less profound effects involving astigmatism. The magenta ellipsoid is the largest and therefore this sample for the left eyes with the most severe DR had the most variable distribution with respect to the means of the other eyes concerned. This is an important new finding concerning the possible relations between severity of DR and refractive state, although further studies using these methods are needed to confirm this result. After removal of outliers in samples for right or left eyes, the sample size $(n=19)$ for DME subjects was considered too small to proceed with a similar analysis as above.

Results obtained for the mean visual acuities in the 
various groups are represented in the bar chart (Figure 3). From the graph it can be seen that subjects with DR and DME exhibit poorer mean visual and pinhole acuities in both right and left eyes compared to subjects without DME and DR (the higher the bars on the LogMAR scale the worse the visual acuity). As expected, subjects with DME reveal much poorer mean visual and pinhole acuities in both right and left eyes when compared to subjects with DR. An interesting observation to note, when comparing mean distance visual acuity in subjects' left eyes with DME to their mean pinhole acuities, one might expect the pinhole acuities to improve. However, in this case it remains approximately the same. A possible explanation for this result is that in this sample the average prevalence of more severe DME was found in subjects' left eyes, causing insignificant improvements in the mean acuity. For the right eyes, the expected improvements were seen but the severity of DME was less on average.

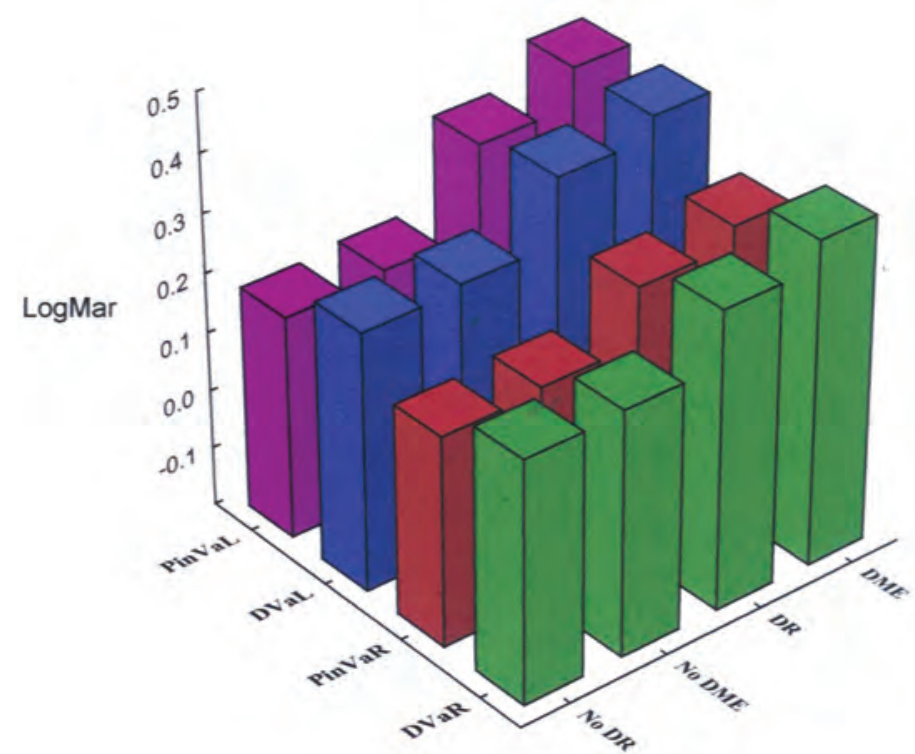

Figure 3. LogMAR bar graph representing the mean distance (DVa) and pinhole visual acuities (PVa) in subjects right (R) and left (L) eyes with and without DR and DME. A LogMAR reading of 0 indicates $6 / 6$ visual acuity, and 0.5 indicates visual acuity of just less than $6 / 18$. Thus the larger any bar the worse the visual acuity. With DR or DME visual acuities were worse than without DR or DME irrespective of type of visual acuity (habitual or pinhole) measured or laterality (whether right or left eyes were measured).

Error plots in Figure 4 were generated to compare the mean contrast visual acuity levels. Subjects with DR displayed much lower acuities on all three levels of CVA in left eyes. No significant differences were apparent at the $100 \%$ contrast levels. A significant difference in CVA2.5\% level was observed for both right $(t(200)=-2.0, p=0.042)$ and left eyes $(t(67)$, $p=0.035)$ of the DR subjects. In subjects with DME, significant differences in means for both CVA $10 \%$ $(t(200)=-2.3, p=0.021$ in right eyes, and $t(200)=$ $-2.0, p=0.046$ in left eyes) and CVA2.5\% ( $t$ (200) $=-2.2, p=0.033$ in right eyes, and $t(200)=-2.0$, $p=0.045$ in left eyes) levels were seen. It can therefore be concluded that there are indications that even small deteriorations in contrast visual acuities at the 2.5\% level might signify the presence of DR and larger differences in contrast acuities at both the $10 \%$ and the $2.5 \%$ might be indicative of DME. The ETDRS contrast sensitivity charts might thus be a simple and fast but effective procedure to identify early or subtle levels of injury to the retina or macula itself (but further investigations in this area are suggested to explore this issue and confirm the result).

Results from the Farnsworth D15 tests revealed a higher percentage of tritan colour defects in the majority of DM subjects. However, no statistically significant differences were found between tritan colour defects and the presence or absence of DR and/or DME.

A range of between $20 \%$ to $24 \%$ of subjects with DR and DME reported central visual field defects on the Amsler grid respectively. The Amsler grid is a useful and simple test that is effective for self-monitoring of the integrity of the central vision field should DME develop. This suggests the need to routinely dispense these charts, or similar ones which can be generated with common computer software, with complete instructions to all diabetics attending the clinic.

The mean recommended IOP range ( $16-18 \mathrm{mmHg})$ was observed in all subjects and showed no correlation between those subjects with and without DR and DME.

Subjects with DR reported significant differences in mean scores in the question category regarding consumer and social interactions $(t(200)=-2.159, p$ $=0.032$ ). Subjects with DME reported significant differences in categories of leisure and work $(t$ (200) $=$ $-2.597, p=0.010)$, consumer and social interactions $(t(200)=-2.239, p=0.026)$, and emotional reaction to vision loss $(t(27.8)=-2.511, p=0.018)$. This indicates that these subjects reported difficulty in performing activities or tasks in these domains mainly 
due to their visual impairment.

Subjects with DME on average reported having their last eye examinations at 29.4 months ago, and subjects with DR 24 months ago.

(a) Right Eyes

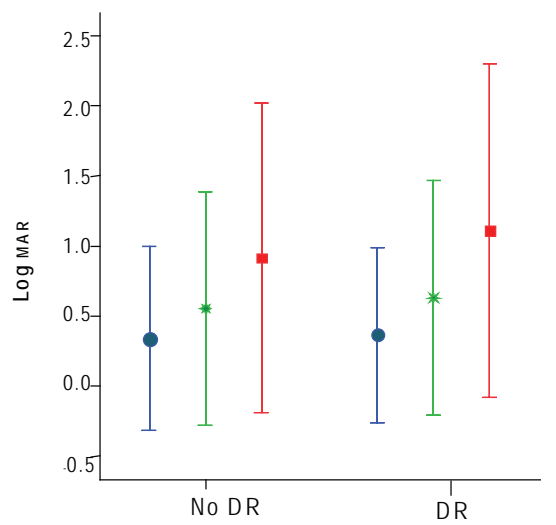

(c)

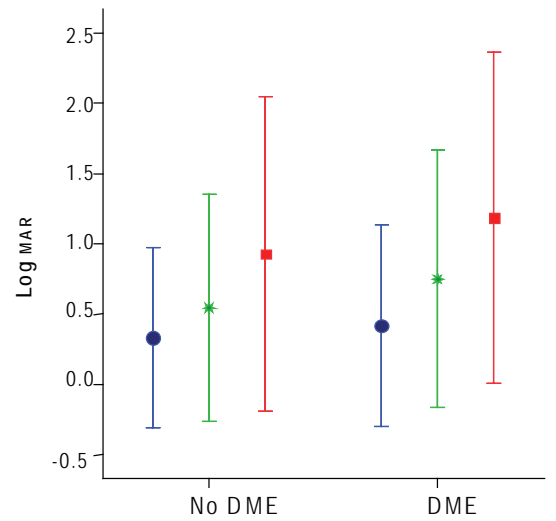

\section{Discussion and Conclusion}

There are very few studies in South Africa regarding visual or ocular aspects in DM, DR and DME. Therefore, the results of visual parameters assessed

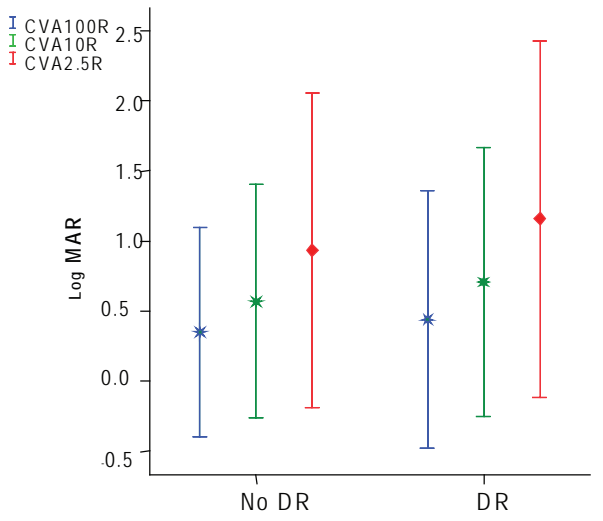

(d)

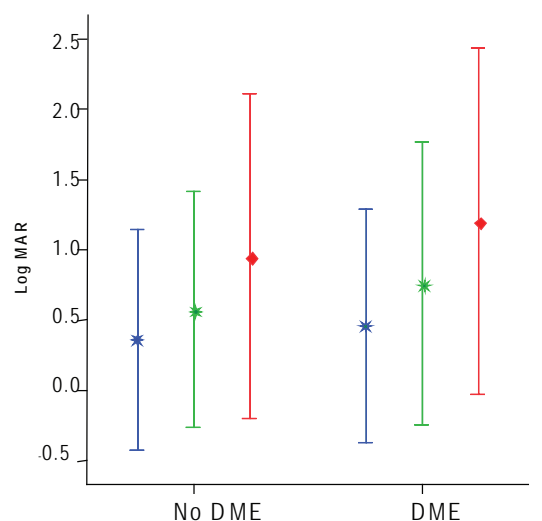

Figure 4. Error plots representing contrast sensitivity levels in subjects right and left eyes with and without DR (a and b), and with and without DME (c and d). Symbols (filled circles, asterisks or squares) represent the mean LogMAR acuity of the sample, and whiskers represent \pm 2 SEM. Blue represents stimuli with higher contrast ( $100 \%$ or basically black-on-white) while green and red represents stimuli with lower levels of contrast (10 and 2.5\% respectively).

in this study provide information in this regard and a more complete clinical analysis of visual aspects in DM, DR, and DME.

The prevalence results indicate that approximately 1 in 5 and approximately 1 in 8 DM persons under treatment presented with DR and DME at this clinic respectively. Prevalence in other diabetic clinics in South Africa includes, Joannou et al ${ }^{13}$ who reported a slightly larger prevalence of $30.6 \%$. Similarly, Carmichael et al reported a prevalence of DR of $30 \%$. They found $12 \%$ of DR was severe enough to warrant referral. As a group, they too found that Blacks had significantly less DR than Indians or Whites ${ }^{14}$. In Kalk et al ${ }^{15}$ the prevalence of DR was found to be similar in Blacks, 37\%; Whites; 41\% and Indians; $37 \%$. Only one study conducted in Cape Town reported a prevalence of $55.4 \%$ of DR and $15.6 \%$ of PDR in 243 Black diabetes subjects recruited from three of the largest diabetes clinics in Cape Town ${ }^{16}$. Two retrospective studies were conducted in Kwa-Zulu Natal, one in urban Durban ${ }^{17}$ and one in rural Hlabisa ${ }^{18}$. In the 216 Black and Indian subjects, Motala et al reported a prevalence of $53.2 \%$ of DR in Type $1 \mathrm{DM}$, and $64.5 \%$ of DR in Type $2 \mathrm{DM}^{17}$. In the Hlabisa study, 
the prevalence of DR was $40.3 \%$ of which $11.1 \%$ was severe enough to warrant laser photocoagulation, and $16.2 \%$ were prevalent with $\mathrm{DME}^{18}$. The Barbados Eye Study is the only international study containing a similar mixed cohort. In their study ${ }^{21}$ they reported prevalence of DR in Coloureds (of 19.8\%), Blacks (28.5\%) and Whites (7.5\%), and DME was prevalent in $8.6 \%$.

The significant association of duration of DM found in this study is consistent with the majority of other studies, indicating that with longer duration of DM, DR and DME become more common ${ }^{14,20-21}$.

The above South African studies indicate that systolic BP is significantly associated with DR and/or $\mathrm{DME}^{15,16}$. This is in contrast with our study, where only diastolic BP had a positive association with DR and DME. However, our result is consistent with most international studies involving majority Type 1 DM subjects ${ }^{22}$.

$\mathrm{HbA1c}$ values were a significant risk factor for DR and DME in the majority of studies. However, no apparent associations were found in this study. Possible explanations resulting in this inconsistency could be due to the relatively small DR and DME sample sizes, comparable $\mathrm{HbA} 1 \mathrm{c}$ values between subjects without and with DR and DME and the relatively shorter duration of this study compared to other studies where long-term glycemia control was compared to the presence of DR and DME.

This study provides some evidence that possibly an increase in variation of refractive status and myopia is found in eyes with more severe DR and DME. The 10-year longitudinal Beaver Dam Eye Study found a hyperopic shift in subjects with DR (they equated refractive status to the nearest spherical equivalent refraction and thus did not use the multivariate methods of our study) and found a weak relation of refractive status to baseline readings ${ }^{23}$. The Visual Impairment Project defined myopia as a best corrected minus spherical equivalent power $<-0.50 \mathrm{D}$ and did not find a significant correlation between myopia and $\mathrm{DR}^{24}$.

The results of decreasing VA with increasing severity of DR and DME, as found in this study are consistent with other studies, that is, subjects with more severe DR and DME displayed worse VA ${ }^{25-27}$. Levitt et al $^{16}$ is the only other study in this country, besides ours, that evaluated pinhole distance acuities. However, they used a conventional Snellen chart whereas we used the Lighthouse ETDRS charts. Approximately, fifty three percent were reported to have bilateral moderate VA (defined as 6/12 to 6/36) in their study. In our study, subjects with DR and DME displayed mean pinhole acuities between 6/9.5 and 6/15.

Unfortunately, due to the wide variety of measurement procedures, equipment, and calibrations used for the detection of CVA in various studies, no direct or simple comparison of results can be readily made. However, results from this study show that there are indications that contrast visual acuity can distinguish subjects with DR and DME. CVA in both the $10 \%$ and 2.5\% levels were more sensitive to substantial retinal changes in DME.

Results regarding quality of life from this study can only be directly compared to that of Lamoureux, Hassel and Keeffe who also investigated DR with the IVI profile. Their results are consistent with this study, with the highest restriction of participation also found in the domains of leisure and work, mobility, and consumer and social interactions domains ${ }^{28}$. These results indicate the need for suitable rehabilitation programmes in these areas for diabetic subjects especially for those with DR and DME in order to improve their quality of life. For example, in the mobility domain possible interventions could include marking of stair edges with white paint and orientation training and in leisure and work it might include the provision of adaptive devices. For example, if one of the main visual needs is simply being able to read a newspaper or to read insulin dosages, ruler type magnifiers or clip-on magnifiers can be prescribed. For monitoring blood glucose levels, meters with large print displays or audible output can be made available.

One possible limitation of this study is that it is clinic based. Therefore, the differences in gender and ethnic distribution are possibly caused by socio-economic factors. Thus, the prevalence and associations of DR and DME as calculated in this study do not allow for accurate estimates of DR and DME in the general population although they might have some predictive value. The results of this study are therefore probably mainly applicable to typical diabetic clinics in urban areas of South Africa.

A second limitation is that DR and DME subjects only consisted of a small percentage of the total sample. Larger sample sizes of these specific groups would be useful in the determination of more conclu- 
sive relationships between certain variables and DR and DME. Other temporal aspects such as the time of day when measurements were obtained, fatigue and lack of concentration are also dependent on glucose levels that fluctuate throughout the day, and this might have had important influences on some measurements obtained during this study. These limitations of this study, however, provide various areas or topics that need to be addressed with further research.

Results from this study can be used by health-care personnel to form demographic, medical and visual profiles which can aid to help identify DR and DME in high-risk individuals. Additionally, these results can contribute to the implementation of various programmes and strategies that will aid in earlier identification and better management of DM. Research in these areas, in both South Africa and elsewhere, is quite limited and thus it was hoped that this research could contribute towards better understanding and perhaps suggest important areas for future investigation or further development.

\section{Acknowledgements}

To Doctor VB Holland, staff, and the patients of Helen Joseph Hospital in Johannesburg, and Doctors Imraan R Munshi and Shashikant Kassen for their contributions towards diagnosing the fundus photographs.

\section{References}

1. Wild S, Roglic G, Green A, Sicree R, King H. Global prevalence of diabetes. Diabetes Care 200427 1047-1051.

2. Verma L, Gunjan P, Terwari HK, Gupta SK, Murthy GVS, Sharma Nisha. Screening for diabetic retinopathy by nonophthalmologist: an effective public health tool. Acta Ophthalmol Scand 200381 373-377.

3. Lightstone A. Review of the effectiveness of optometrists in the monitoring of diabetic retinopathy. Public Health Research Report 95, Essex, 1999.

4. Sacharowitz H. Visual impairment in South Africa: achievements and challenges. S Afr Optom 200564 139-149.

5. Porta M, Allione A. Current approaches and perspectives in the medical treatment of diabetic retinopathy. Pharmacol Ther 2004103 167-177.

6. Ciulla TA, Amador AG, Zinman B. Diabetic retinopathy and diabetic macular edema: Pathophysiology screening and novel therapies. Diabetes Care 200326 2653-2668.

7. Gardner TW, Antonetti DA, Barber AJ, and The Penn State Retina Research Group. Diabetic retinopathy: more than meets the eye. Surv Ophthalmol 200247 S253-262.
8. Willkinson CP, Ferris FL, Klein RE, and the Global Diabetic Retinopathy Project Group. Proposed International Clinical Diabetic Retinopathy and Diabetic Macular Edema Disease Severity Scales. Ophthalmology 2003110 1677-1682.

9. Weih LM, Hassel JB, Keeffe J. Assessment of the Impact of Vision Impairment. Invest Ophthalmol Vis Sci 200243 927-935.

10. de Boer MR, Moll AC, de Vet HCW, Terwee CB. Psychometric properties of vision-related quality of life questionnaires: a systematic review. Ophthalmic Physiol Opt 2004; 24 257-273.

11. Harris WF. Representation of dioptric power in Euclidean 3-space. Ophthalmic Physiol Opt 199111 130-136.

12. Harris WF, Malan DJ, Rubin A. The distribution of dioptric power: ellipsoids of constant probability density. Ophthalmic Physiol Opt 199111 381-384.

13. Joannou J, Kalk WJ, Mohammed I. Screening for diabetic retinopathy in South Africa with a $60^{\circ}$ retinal colour photography. J Intern Med 1996239 43-47.

14. Carmichael T, Carp GI, Welsh ND, Kalk WJ. Effective and accurate screening for diabetic retinopathy using a $60^{\circ}$ mydriatic fundus camera. S Afr Med J 200595 57-61.

15. Kalk WJ, Joannou J, Ntsepo S, Mahomed I, Mahanlal PM, Becker PJ. Ethnic differences in the clinical and laboratory associations with retinopathy in adult onset diabetes: studies in patients of African, European and Indian origins. $J$ Intern Med 1997241 31-37.

16. Levitt NS, Bradshaw D, Zwarenstein MF, Bawa AA, Maphumolo S. Audit of public sector diabetes care in Cape Town, South Africa: high prevalence of complications, uncontrolled hyperglycaemia and hypertension. Diabet Med 199714 1073-1077.

17. Motala AA, Pirie FJ, Gouws W, Amod A, Omar MAK. Microvascular complications in South African patients with long duration diabetes mellitus. S Afr Med J 200191 987-991.

18. Rotchford AP, Rotchford KM. Diabetes in rural South Africa- an assessment of care and complications. S Afr Med J 200292 536-541.

19. Leske MC, Wu SY, Hyman L, and Barbados Eye Studies Group. Diabetic retinopathy in the black population- the Barbados Eye Study. Ophthalmology 1999106 1893-1899.

20. Klein R, Klien BE, Moss SE, Cruickshanks KJ. The Wisconsin Epidemiological Study of Diabetic Retinopathy: XVII. The 14-year incidence and progression of diabetic retinopathy and associated risk factors in type 1 diabetes. Ophthalmology 1998105 1801-1815.

21. Leske MC, Wu SY, Hyman L, Li X, Hennis A, Connell AMS, Schachat AP. Hyperglycemia, blood pressure and the 9-year incidence of diabetic retinopathy- the Barbados Eye Study. Ophthalmology 2005112 799-805.

22. Aiello LP, Cahill MT, Wong JS. Systemic considerations in the management of diabetic retinopathy. Am J Ophthalmol 2001132 760-776.

23. Lee KE, Klein BE, Klein R, Wong TY. Changes in refraction over 10-years in an adult population: The Beaver Dam Eye Study. Invest Ophthalmol Vis Sci 200243 2566-2571. 
24. McKay R, McCarty CA, Taylor HR. Diabetic retinopathy in Victoria, Austrialia: the Visual Impairment Project. Br J Ophthalmol 200084 865-870.

25. Saaddine J, Benjamin S, Pan L. Prevalence of visual impairment and selected eye diseases among persons aged $>$ 50 years, with and without diabetes, United States, 2002.

26. Morbidity and Mortality Report 200453 1069-1071.

Rema M, Ponnaiya M, Mohan V. Prevalence of retinopathy in non insulin dependent diabetes mellitus at a centre in Southern India. Diabetes Res Clin Pract 199634 29-36.

27. Moss SE, Klein R, Klein BE. The 14-year incidence of visual loss in a diabetic population. Ophthalmology 1998105 998-1003.

28. Lamourex E, Hassell JB, Keeffe JE. The impact of diabetic retinopathy on participation in daily living. Arch Ophthalmol 2004122 84-88. 